\title{
Computation of fluid flow and pore-space properties estimation on micro-CT images of rock samples
}

\author{
M. Starnoni ${ }^{\mathrm{a}, *}$, D. Pokrajac ${ }^{\mathrm{a}}$, J. E. Neilson ${ }^{\mathrm{b}}$ \\ ${ }^{a}$ School of Engineering, University of Aberdeen, King's College, Aberdeen AB24 3UE Scotland, United Kingdom \\ ${ }^{b}$ Department of Geology and Petroleum Geology, University of Aberdeen, King's College, Aberdeen AB24 $3 U E$ Scotland, \\ United Kingdom
}

\section{Abstract}

Accurate determination of the petrophysical properties of rocks, namely REV, mean pore and grain size and absolute permeability, is essential for a broad range of engineering applications. Here, the petrophysical properties of rocks are calculated using an integrated approach comprising image processing, statistical correlation and numerical simulations. The Stokes equations of creeping flow for incompressible fluids are solved using the Finite-Volume SIMPLE algorithm. Simulations are then carried out on threedimensional digital images obtained from micro-CT scanning of two rock formations: one sandstone and one carbonate. Permeability is predicted from the computed flow field using Darcy's law. It is shown that REV, REA and mean pore and grain size are effectively estimated using the two-point spatial correlation function. Homogeneity and anisotropy are also evaluated using the same statistical tools. A comparison of different absolute permeability estimates is also presented, revealing a good agreement between the numerical value and the experimentally determined one for the carbonate sample, but a large discrepancy for the sandstone. Finally, a new convergence criterion for the SIMPLE algorithm, and more generally for the family of pressure-correction methods, is presented. This criterion is based on satisfaction of bulk momentum balance, which makes it particulary useful for pore-scale modelling of reservoir rocks.

Keywords: Digital rock physics, Permeability estimation, Carbonate, Finite-Volume SIMPLE,

9 Two-point correlation, Micro-Computer Tomography

\section{Introduction}

Fluid flow in porous media is encountered in many practical engineering problems, including oil, water and gas flow in petroleum reservoirs, transport of water and contaminants in unsaturated soils, heating and drying in industrial processes and storage of $\mathrm{CO}_{2}$ in deep underground aquifers.

The importance of pore-scale simulations lays on the fact that fluid properties, geometry of the medium and boundary conditions are much easier to systematically investigate in computer simulations rather than experiments, and that we can use the results obtained from pore-scale simulations to reduce uncertainties in predicting larger-scale phenomena. Advances in pore-space visualization to very high image resolution and constant increase in computer power also contributed to the popularity of this field of study. In particular, image acquisition techniques have advanced from statistical reconstruction of threedimensional representations of the pore-space based on two-dimensional images, to three-dimensional images directly produced by micro-Computer Tomography $(\mu$-CT). With modern $\mu$-CT scanners, an 
instrument housing its own source of X-rays, the 3D representation of the pore-space is reconstructed from a series of $2 \mathrm{D}$ projections taken at different angles, allowing for reproducing the exact microstructure of a rock sample down to a resolution of $1 \mu \mathrm{m}$. This enables predictions to be made on many 3D images of small rock samples, providing data that would be much more difficult or impossible to obtain using traditional experiments. A recent review on pore-scale imaging and modelling can be found in Blunt et al. (2013).

There are two ways to compute pore-space properties: network modelling and direct simulations. In network modelling (Bakke et al., 1997; Oren et al., 1998; Blunt et al., 2002; Valvatne et al., 2005), the complex geometry of the medium is replaced by a topologically representative system of larger void spaces called pores, connected by straight channels called throats. In direct simulations instead, the real pore-space of the medium is the solution domain for the governing equations. Lattice Boltzmann methods (LBM) (Frisch et al., 1986; Chen and Doolen, 1998; Zu and He, 2013) are the most popular approach for computing single and multiphase flow directly on pore-space images. LBM has a kinetic nature and consists of a number of particles moving in a regular lattice and undergoing momentumconserving collisions. The resulting fluid properties are then obtained by statistical averaging. LBM can also be seen as a discretization of the Boltzmann equation for a particle velocity distribution field.

Another more traditional direct approach involves solving the Navier-Stokes equations using conventional grid based methods such as finite difference (FD), finite element (FE) and finite volume (FV). Pioneering investigations in this field are due to Adler et al. (1990). They computed permeability values on a fictitious porous medium, sharing the same statistical properties of real Fontainebleau sandstones, and then compared the numerical values with experimental data. They found the predicted permeabilities five times greater than the measured ones, although the general shape of the experimental curve was well-reproduced. Martys et al. (1994) and Coelho et al. (1997) simulated fluid flow and dispersion through random packings of spheres and packings of grains of arbitrary shape respectively, in order to obtain universal curves for fluid permeability. Their data were found to agree well with experimentally obtained permeability values of several sandstones. The first use of $2 \mathrm{D}$ digital images of real porous microstructures, captured using an optical microscope, is due to Tashman et al. (2003). They first validated the numerical scheme by comparing the predicted permeability values of a medium of packed columns to a closed form solution, and then evaluated the permeability tensor of three natural granular microstructures (Glass beads of $1 \mathrm{~mm}$ diameter and two sands with an average particle diameter between 0.40 and $0.60 \mathrm{~mm}$ ). Al-Omari and Masad (2004) computed fluid flow in 3D microstructures of field cores of asphalt mixes, captured using X-rays CT, with average particle diameter between 3 and $10 \mathrm{~mm}$, and compared the predicted permeability values with closed form solutions. Zaretskiy et al. (2010) applied the FE method to study a digitized Fontainebleau sandstone sample, comparing their numerical performance to LBM simulations. Mostaghimi et al. (2013) simulated Stokes flow directly on pore-space images obtained from $\mu$-CT scanning of a range of porous microstructures, including sandstones (mean pore radius 15-20 $\mu \mathrm{m}$ ) and carbonates (mean pore radius 10-15 $\mu \mathrm{m}$ ) (Dong, 2008). They studied the existence and size of the representative elementary volume (REV) (Bear, 1972) showing that the REV for permeability is 
(a)

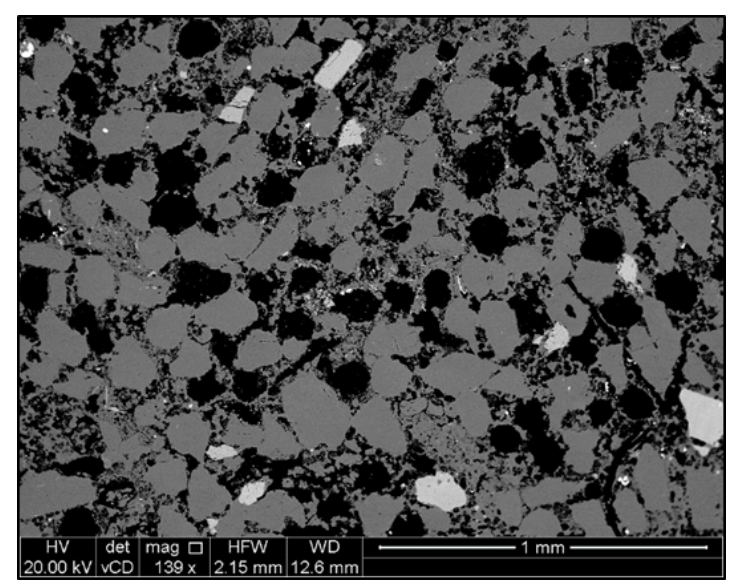

(b)

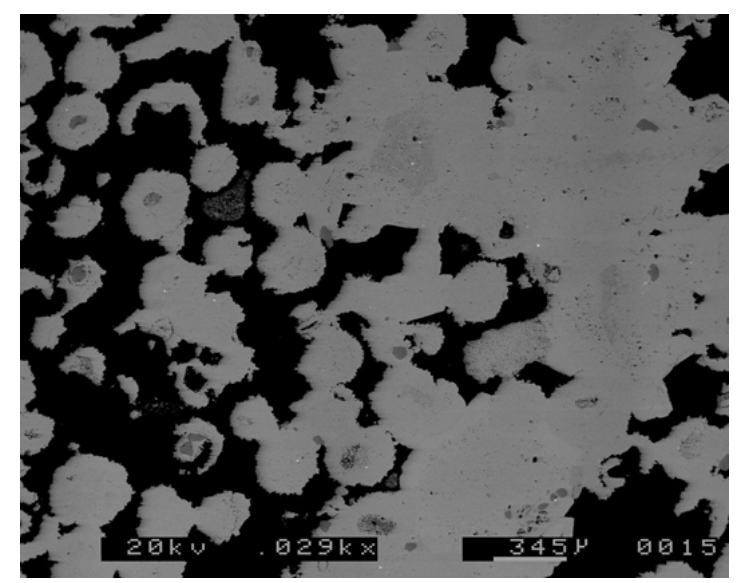

Figure 1. 2D SEM images of the CB sandstone (a) and carbonate (b) rocks

larger than for other static properties, such as porosity. Guibert et al. (2015) investigated the role of mesh sensitivity in computing permeability on a Dausse sandstone, showing that mesh refinement of the original tomographic image has a non-negligible impact on the evaluation of the permeability tensor.

The same approach of using combined high-resolution imaging and numerical simulations has also been used by other researchers in the area of stochastic reconstuction (Øren and Bakke, 2002; Politis et al., 2008) and other fields such as fuel cells (Litster et al., 2013; Sabharwal et al., 2016). In stochastic reconstruction studies, numerical simulations are accompanied by statistical analyses. Statistical analyses offer a valuable tool for characterizing the porous microstructure, and hence are used for assisting in the reconstruction process. However, these studies overall focus on the reconstruction process itself rather than on the characterization of real media. Likewise, fuel cells studies work on the nano-scale, and hence investigate physical processes rather different from those occurring at the micro-scale investigated in digital rock physics. Moreover, they do not simulate Stokes flow, nor explore permeability.

In this paper, we extended the analyses presented in previous studies and developed an integrated approach, comprising rock imaging, statistical spatial correlation and numerical simulations, for thorough characterization of reservoir rocks. Our study is carried out on 3D digital images obtained from $\mu$-CT scanning of two rock formations: one sandstone and one carbonate. The morphological properties of the rocks and the image processing techniques adopted are described in Sec. 2. Then, statistical correlation tools are illustrated in Sec. 3. Next, in Sec. 4, results of single-phase flow simulations are presented, and a comparison of various permeability estimates obtained from different approaches is made. Finally, a novel convergence criterion for the SIMPLE algorithm, and more generally for the family of pressure-correction methods, based on bulk momentum balance, is presented in Sec. 5 .

\section{Rocks description and Image processing}

Core plugs of two rock formations are used in this work: one sandstone (Fig. 1a) and one carbonate (Fig. 1b). Cylindrical cores of the former one $(5 \mathrm{~cm}$ long and $3.75 \mathrm{~cm}$ in diameter) were drilled from a single sample block of Jurassic Lower Calcareous Gritstone Formation from Cayton Bay (CB), Scaraborough (UK). The cores consist mainly of quartz $(76 \%)$, muscovite $(7.5 \%)$ and mitrocline $(6.5 \%)$ 
with a range of Calcite comprising about $4-5 \%$ of the rock mass. Measured porosity and permeability were ranging between $30.5 \%$ and $35 \%$ (average $33 \%$ ) and from 8 to $12 \mathrm{mDa}$ respectively. Porosity was measured by Helium expansion and NMR, while permeability was calculated by injecting low-salinity brine $(1 \mathrm{NaCl})$ through rock cores at room temperature and Confinement pressure of $10 \mathrm{MPA}$, and using Darcy's law (Lamy-Chappuis, 2015). Mean pore diameter observed from Scanning Electron Microscope (SEM) images is $100 \mu \mathrm{m}$. Further details on the morphology of this rock can be found in Lamy-Chappuis et al. (2014).

The second rock formation studied is a carbonate of late Cambrian age. This was cut from an outcrop sample of the Al Bashair Formation, Huqf High, Oman. It is an oolitic grainstone (grains range in size from 200-480 $\mu \mathrm{m}$, median $330 \mu \mathrm{m}$ ) with scattered lime mudstone clasts up to $3 \mathrm{~cm}$ in length ( $<1 \mathrm{~cm}$ thickness). The sample is composed entirely of calcite and, despite its age, contains significant intergranular porosity which has been solution enhanced forming small vugs in places. The intergranular/vuggy pore spaces observed in SEM images range from $90 \mu \mathrm{m}$ to $700 \mu \mathrm{m}$ diameter, together with microporosity $(<20 \mu \mathrm{m})$ within grains (Haines et al., 2015). As with many carbonate samples, the pore system is heterogeneous. Porosity of $15.98 \%$ and permeability of approximately $1500 \mathrm{mDa}$ were measured on a $4.48 \mathrm{~cm}$ in length and 2.53 in diameter core plug. A Coberly-Stevens porosimeter was used to measure the He-porosity on the core plug at ambient temperature and pressure conditions. Heporosity was calculated using the method outlined in Haines et al. (2015, 2016). A Jones Nitrogen permeameter was used to calulate steady-state permeability on the same core plug according to Darcy's Law, by measuring the flow rate of nitrogen through the core plugs at ambient temperature and pressure, using the method described in Haines et al. (2016). A specimen was then manually detached from the core plug and scanned.

CT imaging of the sandstone sample was carried out using a $\mu$-CT scanner housed in the Department of Mechanical Engineering, University of Leeds, at 5 and $10 \mu \mathrm{m}$ pixel resolution (Lamy-Chappuis, 2015). The carbonate sample was scanned using Zeiss XRadia Versa 410 microscope with a 140kV/10W X-rays source housed in the School of Engineering, University of Aberdeen, at pixel resolution of $8.3 \mu \mathrm{m}$ and the field of view covering $16.6 \times 16.6 \times 16.6 \mathrm{~mm}$ (see Fig. 2). Acquisition of $2 \mathrm{D}$ and the reconstruction of 3D images was performed using the software supplied by the respective manufacturers. The latter step involved applying an optimum pixel shift in order to compensate for a slight miss-alignment of the centre of rotation for the sample bottom and top, and an optimum beam hardening constant in order to minimise the effect of beam hardening. Both stages of the process were first done automatically and then checked visually to make sure that the reconstructed pore space does not contain any artefacts. Manual thresholding of the reconstructed 3D images, with threshold level firstly determined using Otsu's method (Otsu, 1975) and then adjusted in such a way to match the experimentally measured porosity, produced 3D binary models consisting of void and solid voxels. A final visual inspection was conducted to verify that the pore space was segmented correctly. Different sub-samples were then extracted from these initial binary images and used as FV meshes. For the sandstone, sub-samples from three different locations were taken (CBsandstone1, 2 and 3) while for the carbonate, sub-samples were extracted by 

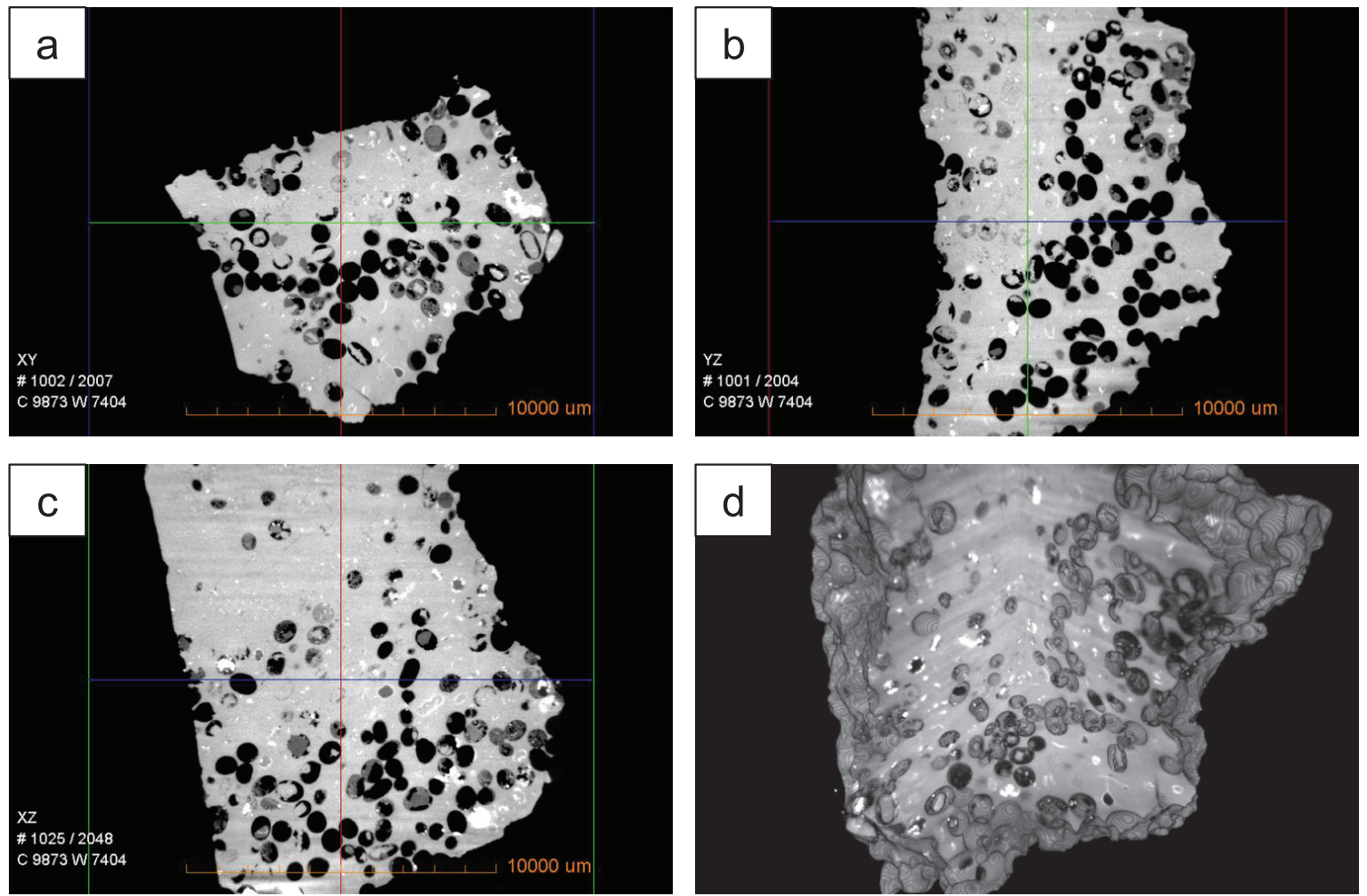

Figure 2. CT imaging of the carbonate sample: $2 \mathrm{D}$ sections corresponding to the three orthogonal planes $(\mathrm{a}, \mathrm{b}$ and $\mathrm{c})$ and the reconstructed $3 \mathrm{D}$ microstructure $(\mathrm{d})$

Table 1. List of samples used in this study

\begin{tabular}{lllll}
\hline Sample & Size $\left[\mathrm{mm}^{3}\right]$ & Resolution $[\mu \mathrm{m}]$ & Voxels & $\phi[\%]$ \\
\hline CBsandstone1a & 1 & 10 & $100^{3}$ & 31.9 \\
CBsandstone1b & 1 & 5 & $200^{3}$ & 32.1 \\
CBsandstone2 & 1 & 5 & $200^{3}$ & 30.9 \\
CBsandstone3 & 8 & 5 & $400^{3}$ & 29.6 \\
Carb200 & 4.6 & 8.3 & $200^{3}$ & 16.5 \\
Carb300 & 15.6 & 8.3 & $300^{3}$ & 15.8 \\
Carb400 & 36.6 & 8.3 & $400^{3}$ & 15.9 \\
Carb500 & 71.5 & 8.3 & $500^{3}$ & 15.8 \\
\hline
\end{tabular}

124 keeping a fixed origin point and considering cubic volumes of increasing size centered at this point.

A list of all the sub-samples considered in this study is given in Table 1 while Fig. 3 shows an example of the reconstructed microstructures for both the rocks.

\section{Two-point spatial correlation function}

A binary indicator function $\xi$ is defined for any position $\mathbf{x}$ in the medium as follows

$$
\xi(\mathbf{x})= \begin{cases}1, & \text { if } \mathbf{x} \text { is in the pore space } \\ 0, & \text { if } \mathbf{x} \text { is in a grain }\end{cases}
$$

229 The two-point correlation function $Z_{2}$ is defined as the probability that two points separated by a distance $\mathbf{r}$ will both lie in the pore space and is mathematically given by (Berryman, 1985; Berryman and Blair, 1986)

$$
Z_{2}(\mathbf{r})=\langle\xi(\mathbf{x}) \xi(\mathbf{x}+\mathbf{r})\rangle
$$


(a)

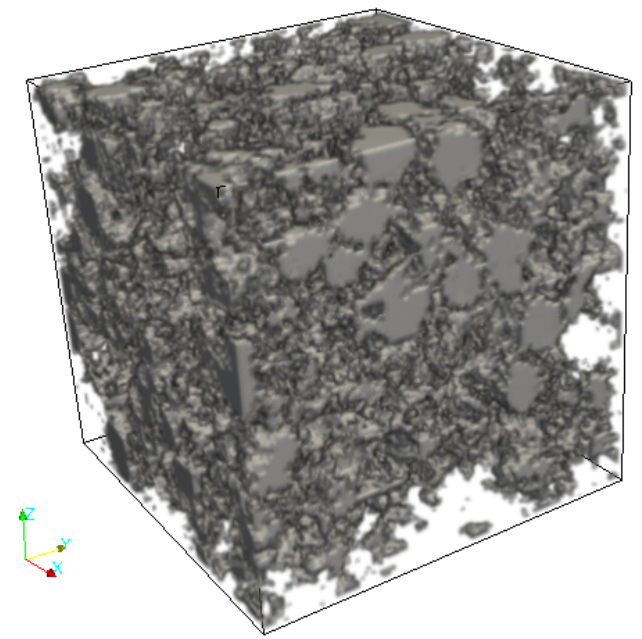

(b)

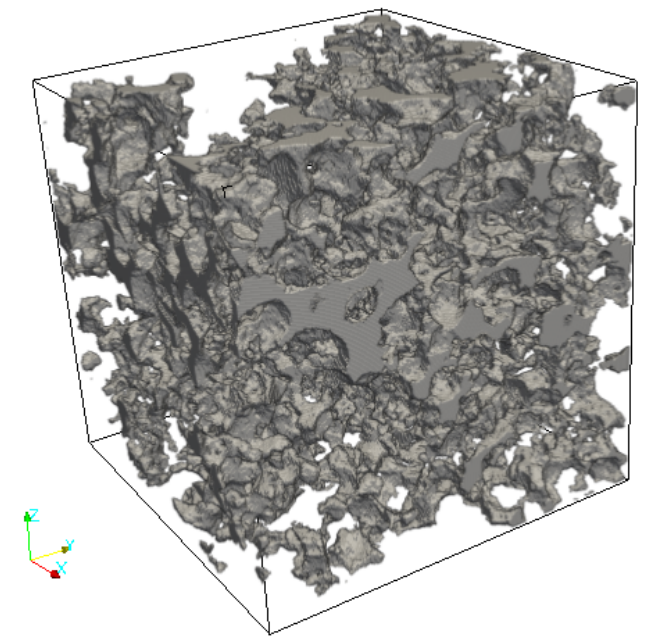

Figure 3. Reconstructed 3D digital images of the samples CBsandstone1a (a) and Carb300 (b)

where angle brackets denote superficial volume averaging over all positions $\mathbf{x}$ and $Z_{2}(0)=\phi$. The twopoint correlation for a 2D digital image is computed using the one-dimensional isotropic method by Berryman (1985). The discrete form of eq. (2) for progressive one-pixel increments $k$ is given by

$$
Z_{2}(k)=\frac{1}{2 k+1} \sum_{l=0}^{2 k} \bar{Z}_{2}(k \cos \varphi, k \sin \varphi), \quad \varphi=\frac{\pi l}{4 k}, \quad 0 \leq k \leq N / 2,
$$

where $N$ is the number of pixels along each side of the image and the discrete function $\bar{Z}_{2}(m, n)$ has the form

$$
\bar{Z}_{2}(m, n)=\frac{1}{N_{\max }} \sum_{\substack{1 \leq i \leq i_{\max } \\ 1 \leq j \leq j_{\max }}} \xi_{i j} \xi_{i+m, j+n},
$$

where $i_{\max }=N-m, j_{\max }=N-n$ and $N_{\max }=i_{\max } j_{\max }$. The procedure is schematically illustrated in Fig. 4. The discrete two-point correlation function $\bar{Z}_{2}$ is computed at points located along the perimeters of arcs centered at the reference point. The arcs are delimited by values of $\varphi$ equal to 0 and $\frac{\pi}{2}$ and their radii are equal to the increment $k$. For each increment $k, \bar{Z}_{2}$ is evaluated separately along $(2 k+1)$ directions $\varphi$, and then averaged to compute the one-dimensional isotropic function $Z_{2}$. Each node of the image is in turn used as reference point, and all segments whose endpoint lies within the image are used for computing $\bar{Z}_{2}$. When $\varphi$ takes values other than 0 and $\frac{\pi}{2}$, i.e. the discrete point does not coincide with a lattice node, $\bar{Z}_{2}(m, n)$ is computed using bilinear interpolation from the 4 closest nodes. The extension to the $3 \mathrm{D}$ case is straightforward, with eqs. (3) and (4) which modify as follows

$$
\begin{array}{r}
Z_{2}(k)=\frac{1}{(2 k+1)^{2}} \sum_{l_{1}, l_{2}=0}^{2 k} \bar{Z}_{2}(k \sin \theta \cos \varphi, k \sin \theta \sin \varphi, k \cos \theta), \\
\theta=\frac{\pi l_{1}}{4 k}, \quad \varphi=\frac{\pi l_{2}}{4 k}, \quad 0 \leq k \leq N / 2,
\end{array}
$$




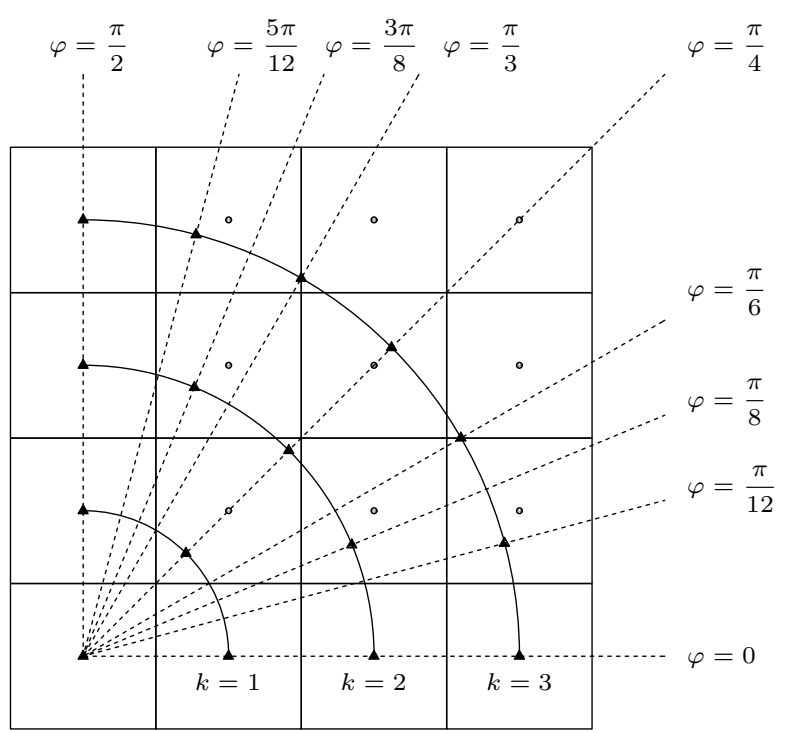

Figure 4. Schematic of the method employed for computing the one-dimensional isotropic two-point correlation function $Z_{2}(k)$ on a $2 \mathrm{D}$ digital image (Berryman, 1985). The discrete two-point correlation function $\bar{Z}_{2}$ is computed at triangular points located along the perimeters of circles centered at the reference point. The circles radii are 1 pixel $(k=1), 2$ pixels $(k=2)$, etc.

where $k_{\max }=N-o, N_{\max }=i_{\max } j_{\max } k_{\max }$ and $\bar{Z}_{2}(m, n, o)$ is computed using trilinear interpolation from the 8 closest nodes when the discrete point does not coincide with a lattice node.

This correlation function has the relevant feature that many physical properties of the porous material, such as porosity $\phi$, pore-grain specific surface area $s$ and effective pore size $r_{c}$, can be obtained from it. Berryman and Blair (1986) showed that

$$
\lim _{r \rightarrow \infty} Z_{2}(\mathbf{r})=\phi^{2}
$$

$$
Z_{2}^{\prime}(0)=-\frac{s}{\chi}
$$

$$
r_{c}=\frac{\phi(1-\phi)}{Z_{2}^{\prime}(0)},
$$

where $\chi$ is 4 for $2 \mathrm{D}$ and 6 for 3D images (Yeong and Torquato, 1998) and the initial slope $Z_{2}^{\prime}(0)$ is computed using ordinary least squares to the first four or five points of the curve. Specific surface area can then be used to estimate the absolute permeability $K$ following the Kozeny-Carman (KC) approach. $\mathrm{KC}$ empirical formulations relate permeability to porosity and hydraulic radius, the latter defined as the ratio of the pore volume and the wetted area. The KC relation developed by Walsh and Brace (1984) is used here:

$$
K=\frac{\phi^{2}}{m_{1} F s^{2}},
$$

where $m_{1}$ is a constant depending on the pore shape ( 2 for circular tubes and 3 for cracks) and $F$ is the electrical formation factor which can be estimated using the relationship given by Archie et al. (1942)

$$
F=\phi^{-m_{2}}
$$

where $m_{2}$ is a constant ranging between 1.8 and 2 for sandstones.

Fig. 5 shows the computed two-point correlation function for the $\mathrm{CB}$ sandstone images. For each sample, four curves are displayed, corresponding to the whole 3D image and three 2D cross-sections 

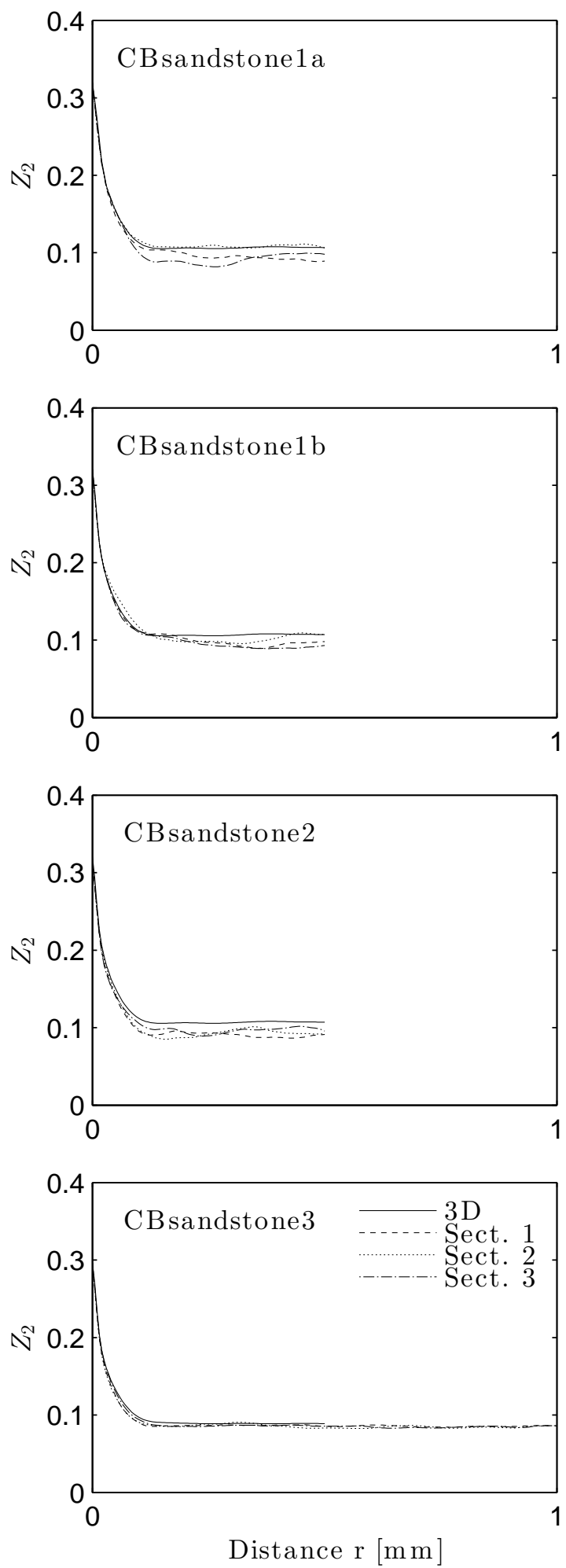

Figure 5. Computed two-point correlation function for the $\mathrm{CB}$ sandstone samples. For each sample, four curves are displayed, corresponding to the whole 3D image (continuous line) and three $2 \mathrm{D}$ cross-sections chosen with the same areal porosity as the volumetric one 
chosen with the same areal porosity as the volumetric one. The $3 \mathrm{D}$ curve is computed using eqs. (5) and (6), while the 2D curves are computed using (3) and (4). The 3D curve smoothly approaches the asymptotic value of $\phi^{2}$ with the same trend for all the samples, suggesting that for this rock $1 \mathrm{~mm}^{3}$ can be assumed as REV for the pore geometry. However, for the smaller samples (CBsandstone1a, 1b and 2, having all volume of $1 \mathrm{~mm}^{3}$ ), the 2D curves behave differently from the 3D one. They all show a distinct minimum corresponding to the mean grain size and then tend to their respective asymptotic value with an oscillatory behaviour. This discrepancy vanishes for the bigger sample (CBsandstone3 having volume of $8 \mathrm{~mm}^{3}$ ), suggesting that $4 \mathrm{~mm}^{2}$ can be assumed as representative area (REA).

The same analysis has been conducted on the carbonate images. The computed two-point correlation fucntions are shown in Fig. 6. As for the $\mathrm{CB}$ samples, the 2D curves show an oscillatory behaviour which is progressively damped with increasing the size of the sample. However, existence of an REV and REA for this rock remains undetermined, due to the significant variability of the carbonate pore system. This heterogeneity is made clearer by plotting the predicted mean pore radius, computed using eq. (9), as a function of the image characteristic size, for all the processed images (Fig. 7). For each sample, 10 values are reported: one for the whole 3D image, and three for each of the three orthogonal directions, i.e. three 2D cross-sections for each direction. For the $\mathrm{CB}$ sandstone a homogenous value in the range of $35-45 \mu \mathrm{m}$ is obtained, corresponding to a mean pore diameter of $70-90 \mu \mathrm{m}$, in agreement with the value of $100 \mu \mathrm{m}$ based on SEM images observations given by Lamy-Chappuis et al. (2014). On the other hand, a larger dispersion is observed for the carbonate. In particular, a drop on the predicted mean pore size is observed for the biggest 3D image. This is due to the heterogenous nature of this rock reflected by the presence of large lithological clusts (see Figure 1b) which were not picked up by the smaller size images. The range of predicted pore radius $<60-130>\mu \mathrm{m}$ corresponds to mean pore diameters of $<120-260\rangle$ $\mu \mathrm{m}$ which falls into the broader interval of $90 \mu \mathrm{m}$ to $700 \mu \mathrm{m}$ observed in SEM images. Furthemore, the mean grain size predicted by the two-point spatial correlation function, i.e. the minimum value of $Z_{2}$ in its initial descending branch, ranging between 240 and $380 \mu \mathrm{m}$, is also in good agreement with the morphological data $(200-480 \mu \mathrm{m})$, obtained using a standard petrogtaphic microscope. This confirms the effectiveness of the method in fully characterizing the pore-system.

In order to assess anisotropy, we sampled the two-point correlation function only following the orthogonal directions, as in Yeong and Torquato (1998) and Øren and Bakke (2002). We than averaged these components to obtain a function for the whole medium, and compared this with the one-dimensional isotropic function computed using the extended Berryman's method for 3D images, eq. (5). Results are shown in Figs. 8 and 9 for the sandstone and carbonate samples respectively. One striking aspect is that sample Carb500 is strongly anisotropic. Fig. 9 clearly shows how the 3 directional functions have all different slope in their initial descending branch. This is the only sample displaying this feature. Another aspect which is worth highlighting is that the average directional $Z_{2}$ is very close to the Berryman's onedimensional isotropic function. The latter has obviously a smoother profile, as sampling is done along multiple directions. However, sampling along multiple directions has a very high computational cost, which makes Torquato's method preferable. 

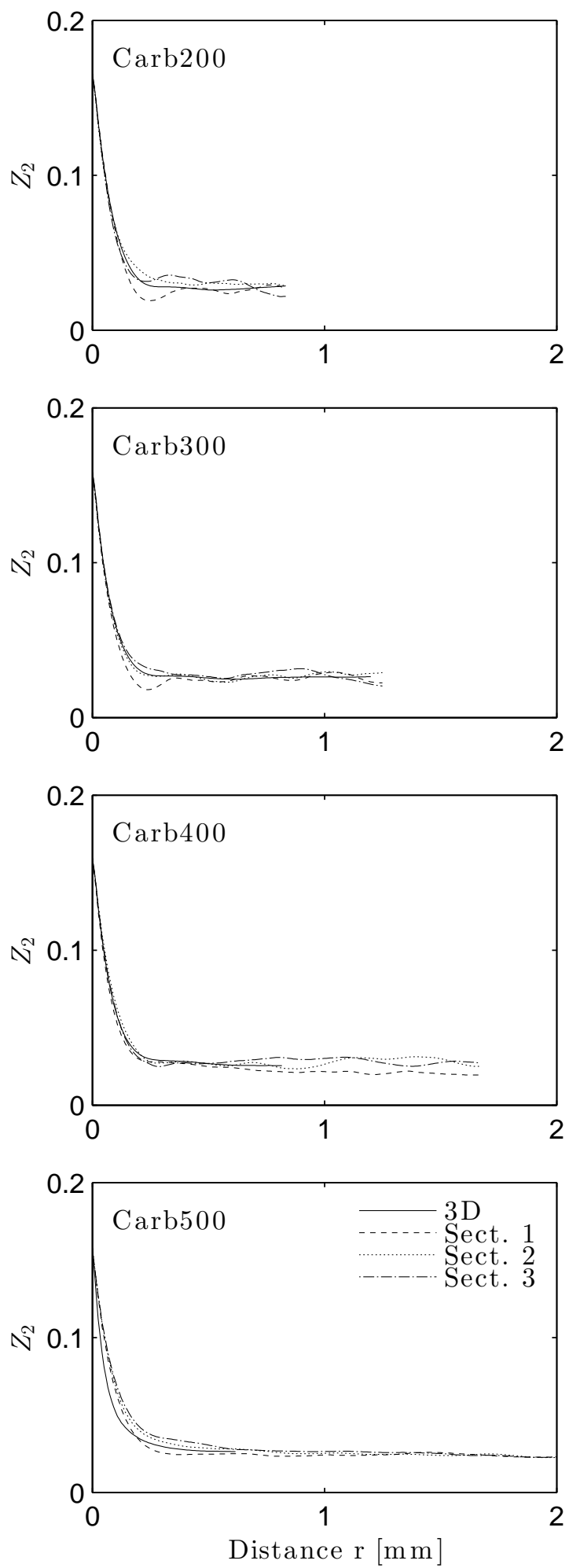

Figure 6. Computed two-point correlation function for the carbonate samples. For each sample, four curves are displayed, corresponding to the whole $3 \mathrm{D}$ image (continuous line) and three $2 \mathrm{D}$ cross-sections chosen with the same areal porosity as the volumetric one 

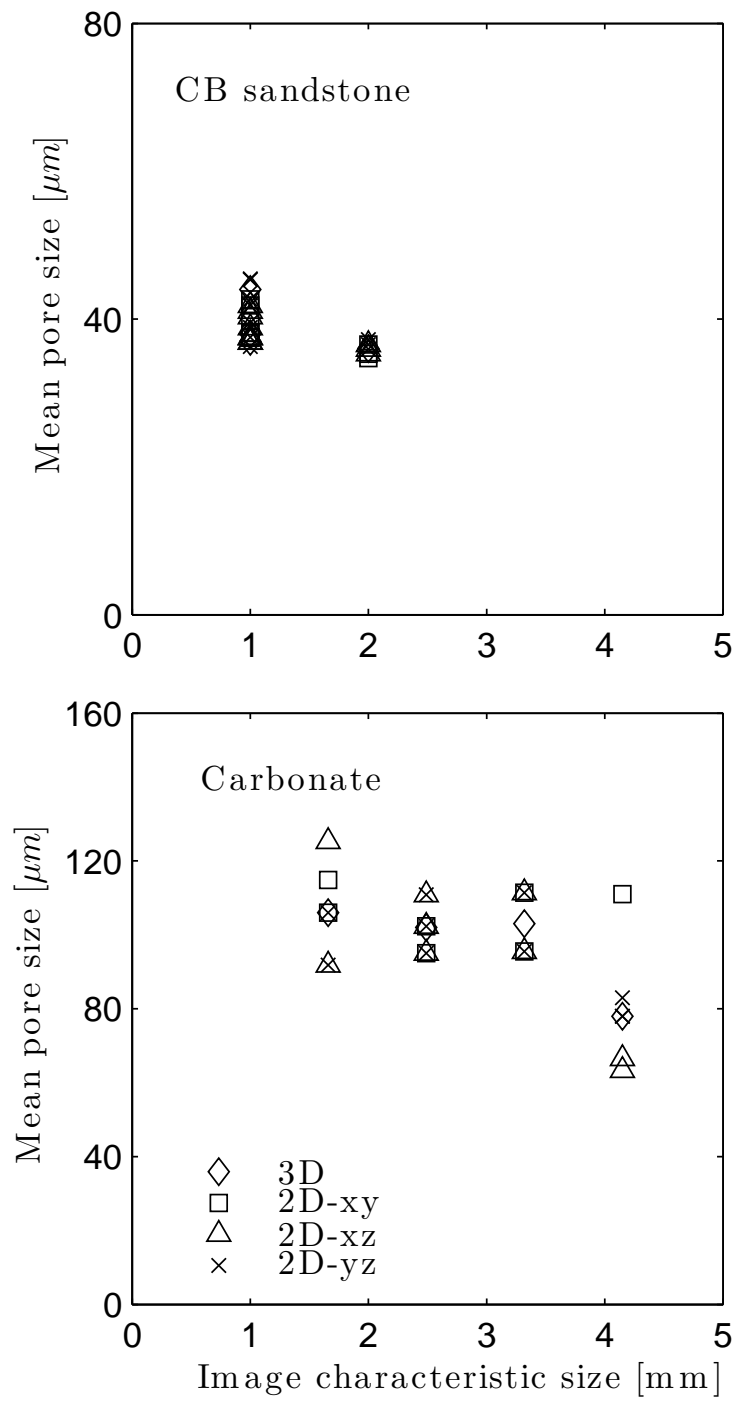

Figure 7. Summary of the estimated mean pore size for all the samples considered in this study. For each sample, 10 values are reported: one for the whole 3D image, and three for each of the three orthogonal planes, corresponding to the three selected 2D cross-sections for each plane 

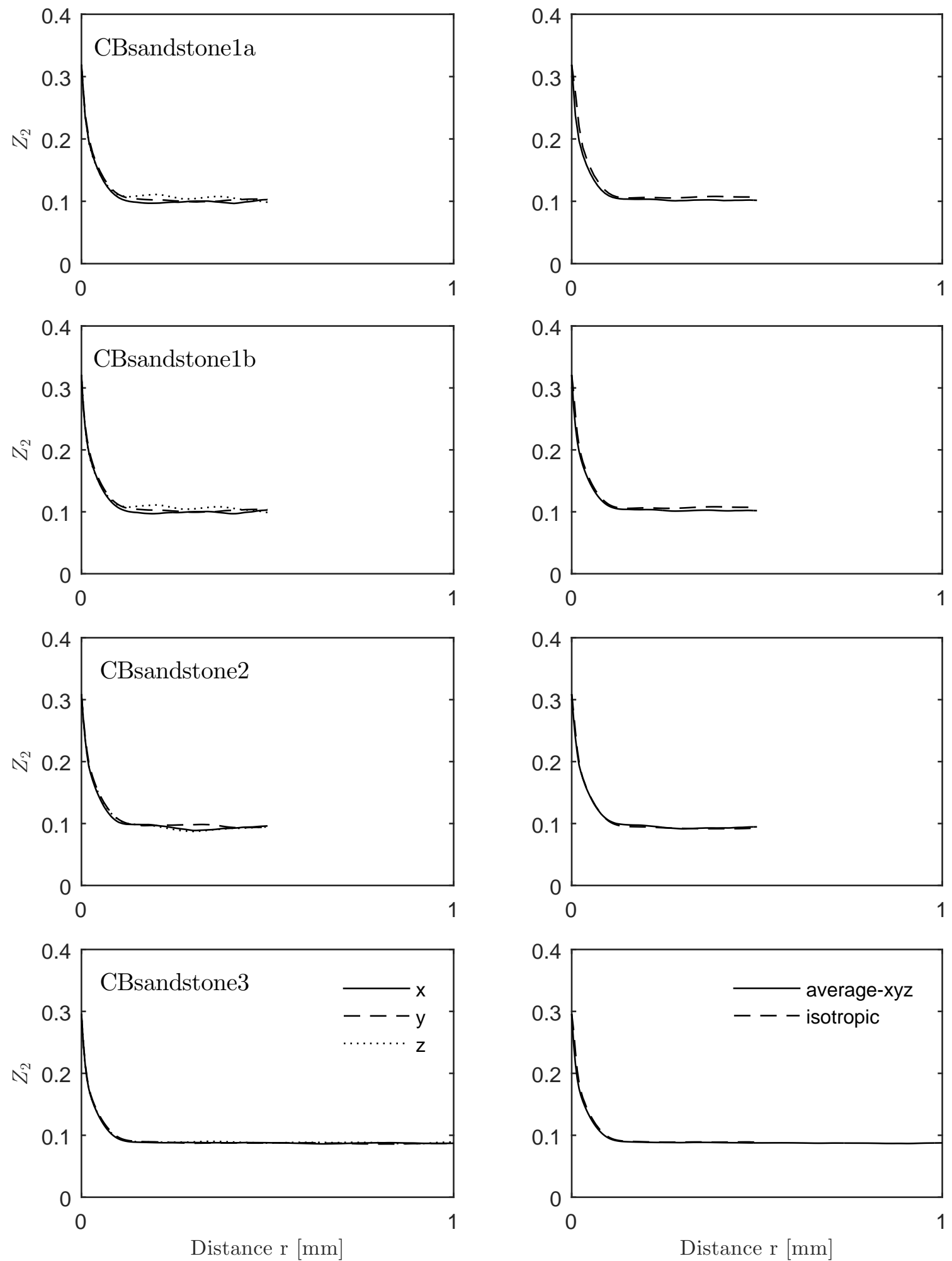

Figure 8. Directional two-point correlation functions (left column) and comparison between the average directional and the 3D isotropic Berrymans's function (right column) for the sandstone samples 

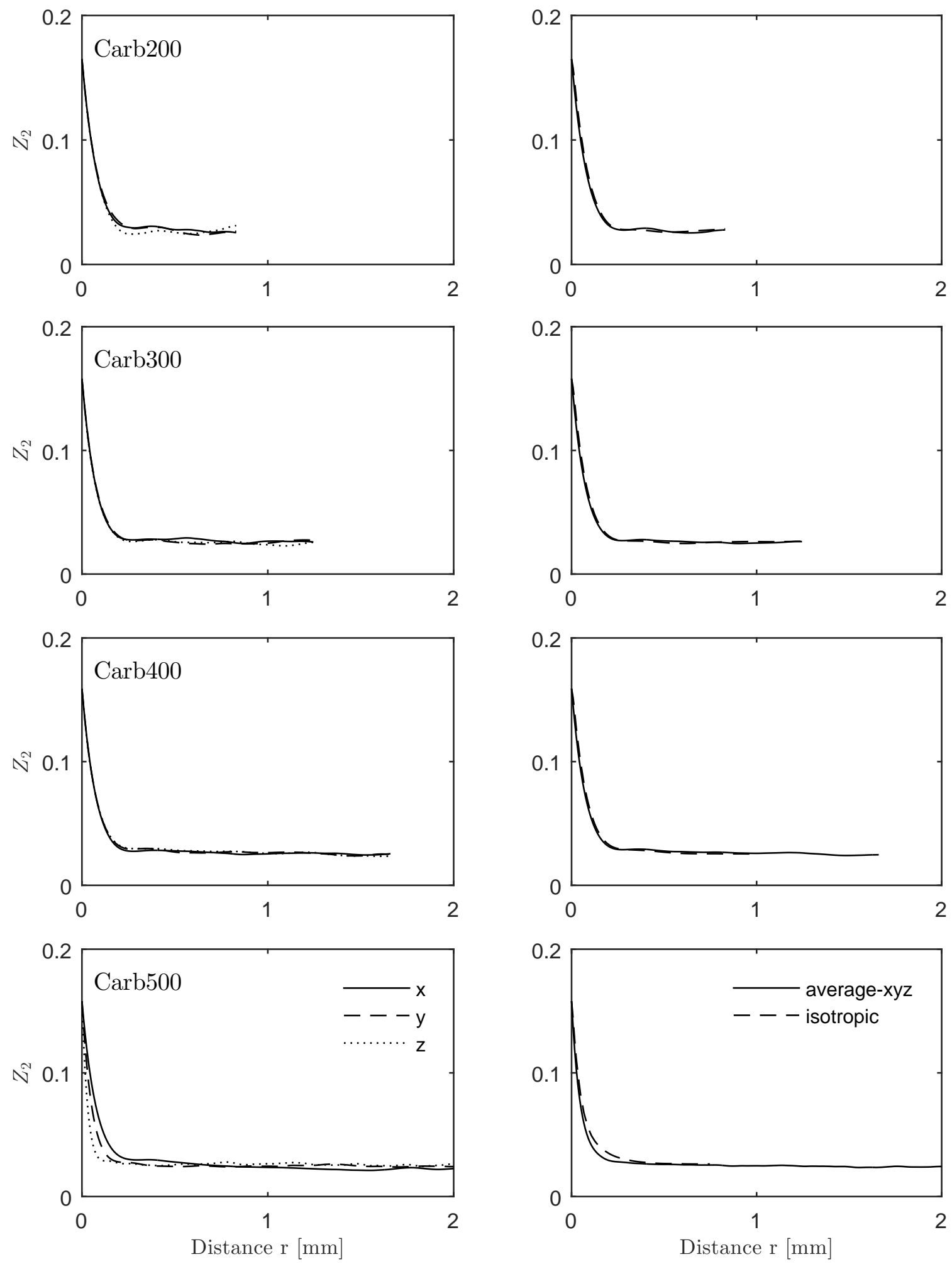

Figure 9. Directional two-point correlation functions (left column) and comparison between the average directional and the 3D isotropic Berrymans's function (right column) for the carbonate samples 
(a)

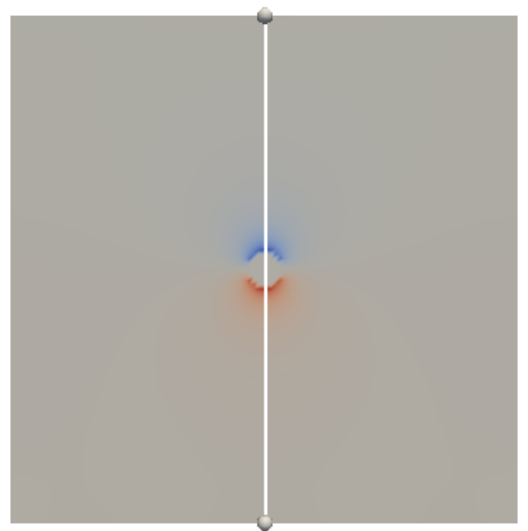

(b)

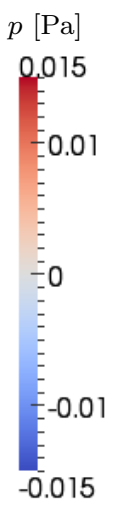

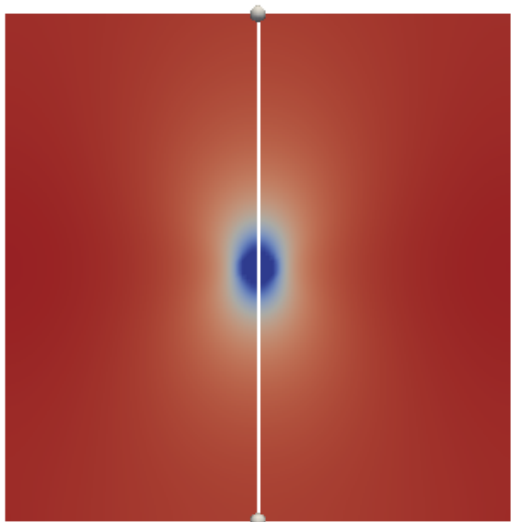

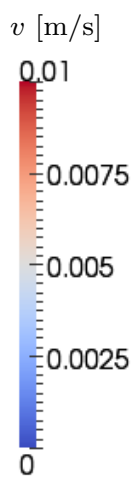

Figure 10. Predicted pressure (a) and velocity component in the direction of the flow (b) on a cross-section parallel to the main flow direction passing through the centre of the sphere. The flow is directed upwards along the depicted line

\section{Computation of fluid flow and permeability prediction}

\subsection{Numerical method}

The Stokes equations of creeping flow for incompressible fluids are written as

$$
\nabla \cdot \mathbf{u}=0
$$

$$
\mu \nabla^{2} \mathbf{u}-\nabla p+\rho \mathbf{g}=0
$$

where $\mathbf{u}$ is the velocity vector, $p$ is the pressure, $\rho$ and $\mu$ are the fluid density and viscosity respectively, and $\mathbf{g}$ is the acceleration of gravity vector.

We solve the incompressible Stokes equations using the FV-SIMPLE algorithm by Patankar (1980) on non-staggered grids. 5 .

\subsection{Validation}

The numerical model has been validated considering the Stokes flow past a sphere benchmark problem. For this test, a fluid of visosity $\mu=0.001 \mathrm{~Pa} \cdot \mathrm{s}$ flowing around a sphere of radius $R=1 \mathrm{~mm}$ with freestream uniform velocity $v_{0}=0.01 \mathrm{~m} / \mathrm{s}$ was considered. The computational domain is a cube of size $2 L^{3}$ centered at the sphere centre, where $L=15 R$ is the distance of the inlet boundary from the centre of the sphere, and was discretized using cubic cells of resolution $R / \Delta x=4$, where $\Delta x$ is the size of the elementary cell. Boundary conditions consist of the freestream uniform velocity at the inlet, prescribed pressure at the outlet and symmetry at the other 4 faces. The numerically evaluated pressure and velocity fields on a cross section passing through the centre of the sphere are shown in Figs. 10a and 10b respectively. Comparison between numerical and analytical values is made in terms of normalized pressure and velocity along the line depicted in Fig. 10. Velocity and pressure are normalized against the freestream uniform velocity $v_{0}$ and the maximum value of pressure at the sphere $p_{\max }=1.5 \mu v_{0} / R$ respectively, while the $\mathrm{x}$-axis, representing the distance $r$ of a point from the centre of the sphere, is normalized against $L$. As Fig. 11 clearly shows, numerical results are in good agreement with the analytical solution, and only small errors can be ascribed to the influence of the boundary conditions and the poor discretization of the sphere. 
(a)

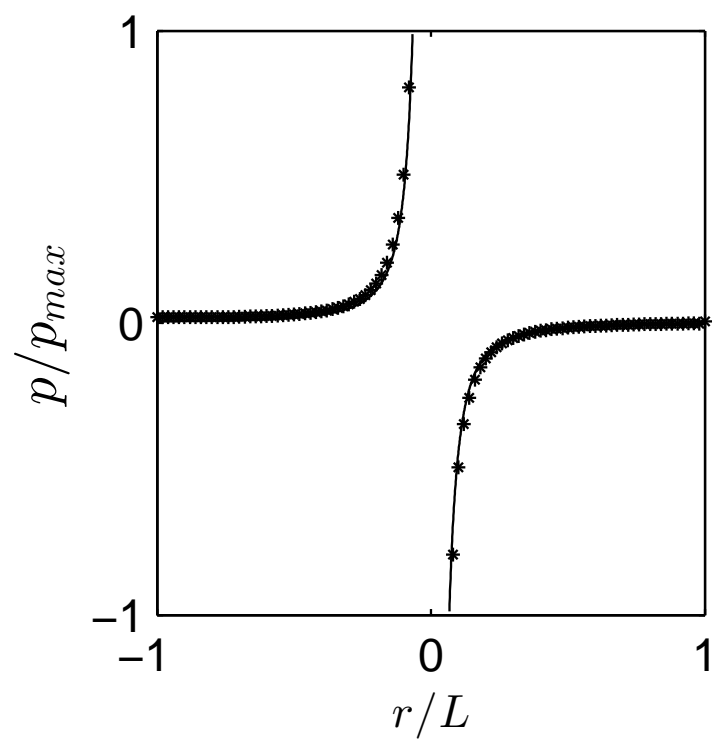

(b)

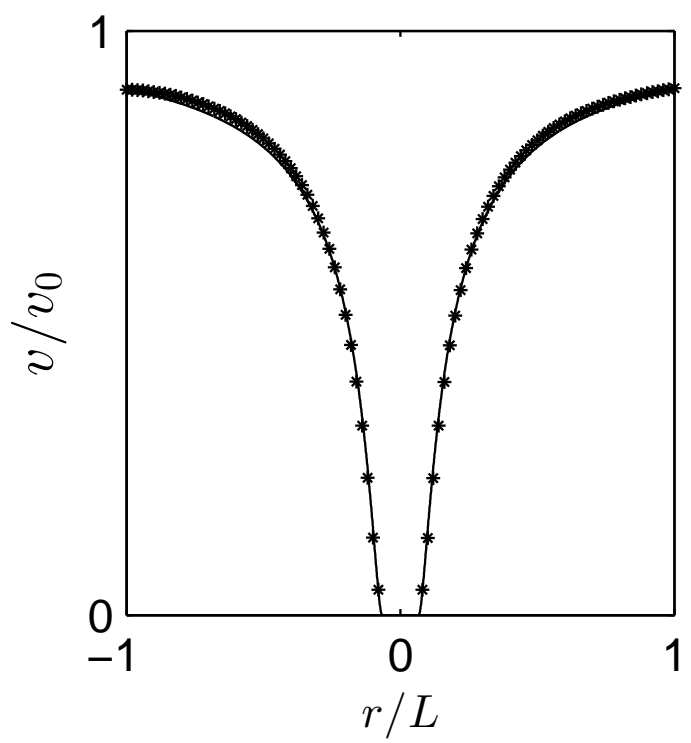

Figure 11. Comparison between analytical and predicted pressure (a) and velocity component in the direction of the flow (b) along the line depicted in Fig. 10. Legend: continuous line is the analytical solution, asteristk marks are the predicted values

\subsection{Results}

We present here results of the simulations carried out on the three dimensional images listed in Table 1. Boundary conditions consist of an applied pressure gradient along the main direction, symmetry at the other four faces and no-slip conditions at the pore-grain interfaces. Internal connectivity of the porous microstructure was assessed before starting the simulations, and isolated dead-end pores (pores that are not connected to the boundary) were set as grains. Furthermore, in order to predict fluid flow more accurately, the original digital images were refined by subdividing each voxel into up to 64 subvoxels. Predicted pressure field and velocity in form of streamlines are shown in Figs. 12 and 13 for the CBsandstone1a and Carb300 samples respectively.

Permeability is then predicted from the computed fluid flow using Darcy's law as follows:

$$
K=\mu \frac{Q L}{A \Delta p}
$$

where $Q$ is the flow rate, $A$ is the area of the face normal to the flow, $\mu$ is fluid viscosity and $\Delta p / L$ is the applied pressure gradient. For a structured cubic grid of characteristic size $L=N \Delta x$ and area $A=N^{2} \Delta x^{2}$, permeability is calculated as

$$
K=\mu \frac{\Delta x}{N} \frac{\sum_{j} u_{j}}{\Delta p}
$$

where $u_{j}$ is the velocity component in the direction of the flow and the sum is over all the pore cells on a plane normal to the flow direction. Constant values of $\mu=0.001 \mathrm{~Pa} \cdot \mathrm{s}$ and $\Delta p=1 \mathrm{~Pa}$ were used in all the simulations.

Anisotropy was also evaluated by running three separate simulations with applied pressure gradient along each of the three orthogonal directions, and then calculating the following anisotropy ratio (Clavaud 
(a)

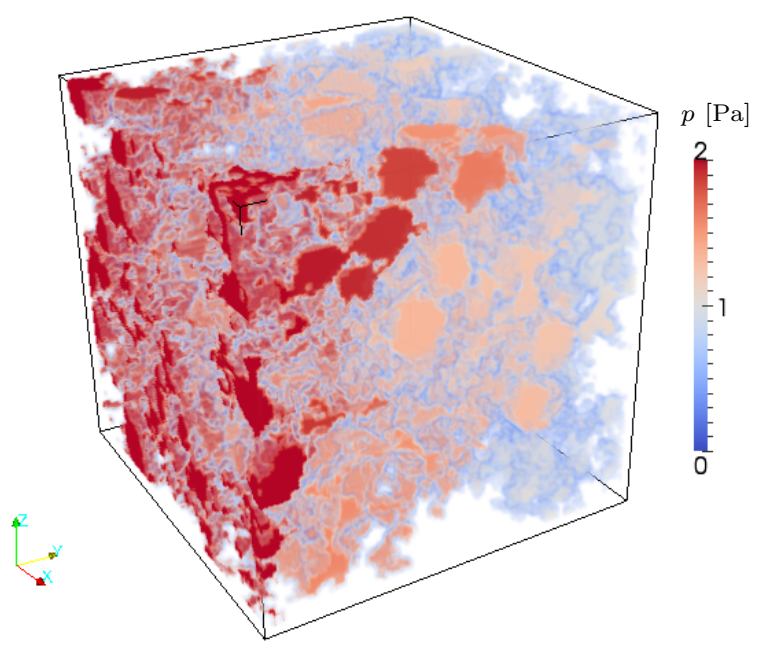

(b)

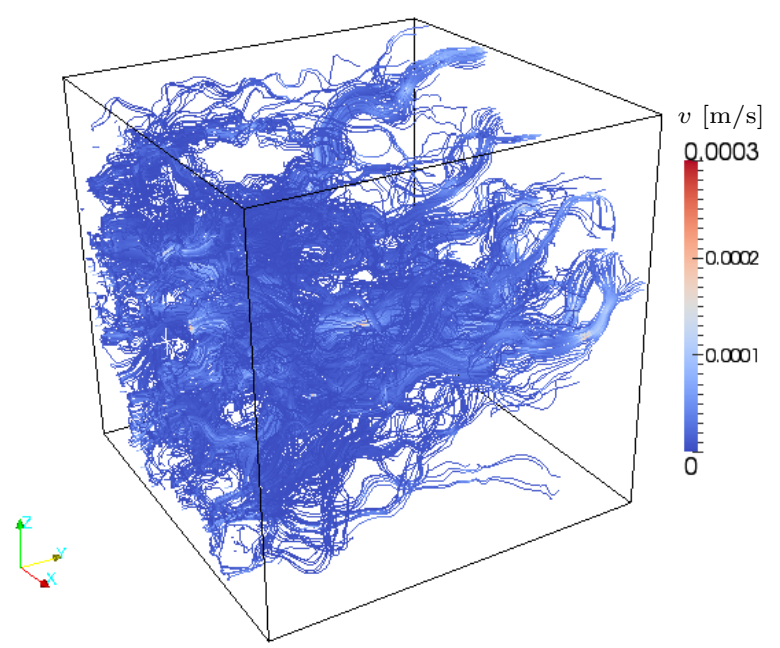

Figure 12. Predicted pressure field (a) and velocity in form of streamlines (b) for the sample CBsandstone1a

(a)

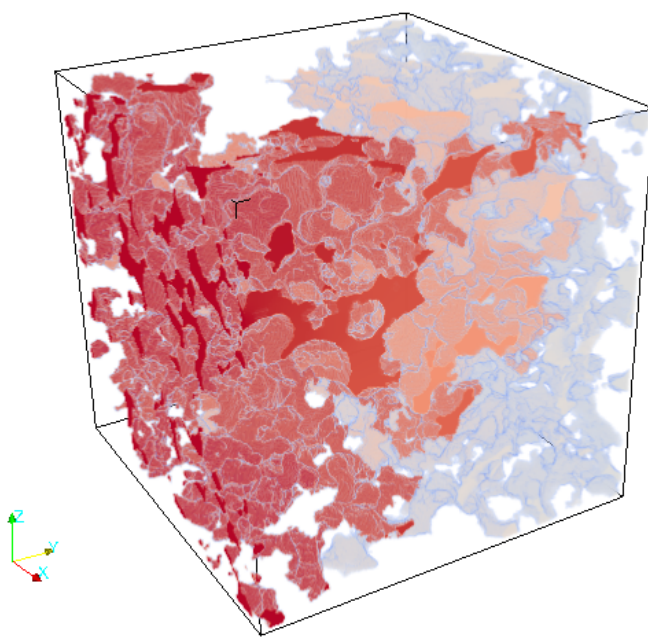

(b)

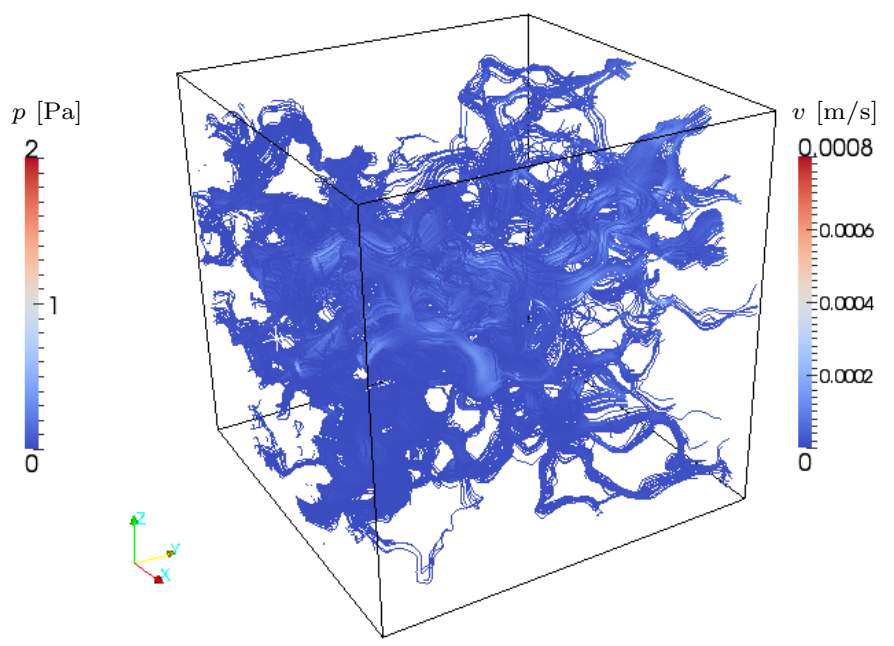

Figure 13. Predicted pressure field (a) and velocity in form of streamlines (b) for the sample Carb300 
Table 2. Predicted permeability for all the samples considered

\begin{tabular}{llllr}
\hline Sample & $K_{x}[\mathrm{Da}]$ & $K_{y}[\mathrm{Da}]$ & $K_{z}[\mathrm{Da}]$ & $\nu$ \\
\hline CBsandstone1a & 1.13 & 1.30 & 0.84 & 0.69 \\
CBsandstone1b & 1.13 & 1.36 & 0.84 & 0.68 \\
CBsandstone2 & 0.87 & 1.06 & 0.85 & 0.88 \\
CBsandstone3 & 0.86 & 1.05 & 0.91 & 0.88 \\
Carb300 & 2.05 & 3.17 & 1.76 & 0.69 \\
Carb400 & 2.99 & 2.70 & 2.76 & 0.94 \\
Carb500 & 0.90 & 1.46 & 0.18 & 0.15 \\
\hline
\end{tabular}

et al., 2008):

$$
\nu=\frac{K_{\min }}{\sqrt{K_{\max } K_{\text {int }}}},
$$

where $K_{\max }>K_{\text {int }}>K_{\min }$ are the three components of the permeability tensor along the three orthogonal directions $x, y, z$, sorted in descending order.

The predicted permeability values are listed in Table 2. For the CB sandstone, permeability remains constant in the narrow range $<0.8-1.2>$ Da with increasing the size of the volume and for different locations of the samples, confirming the homogeneity of the rock and the REV of $1 \mathrm{~mm}^{3} \mathrm{observed}$ using statistical correlation. Anisotropy also disappears for the biggest volume considered. As far as the carbonate is concerned, strong anisotropy is observed for the bigest sample (Carb500) and no clear determination of an REV for this rock can be made. Results in Table 2 confirm the findings reported in previous section, particularly as far as anisotropy of sample Carb500 is concerned.

\subsection{Comparison of different permeability estimates}

We present here a comparison of all the available permeability estimates for the two rock formations considered. Fig. 14 shows the estimated values plotted against the experimentally determined value, the latter measured on the larger core plugs. The FV value has been computed as mean value of the 3 components listed in Table 2 , while the $\mathrm{KC}$ value has been calculated using eq. (10). An estimated value from LBM simulations is also available for the CB sandstone only (pers. comm.).

For the carbonate sample, the measured permeability $(\sim 1500 \mathrm{mDa})$ is roughly twice as much the average FV value of $\sim 850 \mathrm{mDa}$ computed for the largest sample Carb500, while is roughly half the average FV value of $\sim 2800 \mathrm{mDa}$ computed for the smaller Carb400 sample. This inconsistency is probably due to the already highlighted heterogenity of the rock, as well as the difference in scale of the samples, resulting in significant changes in connectivity and tortuosity.

For the CB sandstone, a good agreement between permeability estimates obtained from different numerical approaches (i.e. FV and LBM) is observed. However, divergence up to almost two orders of magnitude appears between the predicted values and the experimentally determined permeability of $\sim 10 \mathrm{mDa}$. Again, this discrepancy can be attributed to the complex microstructure of the rock and the presence of micro-imperfections, i.e. calcite shells fragments (Lamy-Chappuis, 2015), that cannot be detected by the resolution used for scanning the samples. As a result, very intricate pores filled with sub-micro fragments, which in reality have very high pore-grain surface area and very low net pore-volume, are converted into large open pores during the segmentation process, resulting in a much greater predicted permeability than the actual one. The thresholding used for segmentation of the $\mathrm{X}$ - 


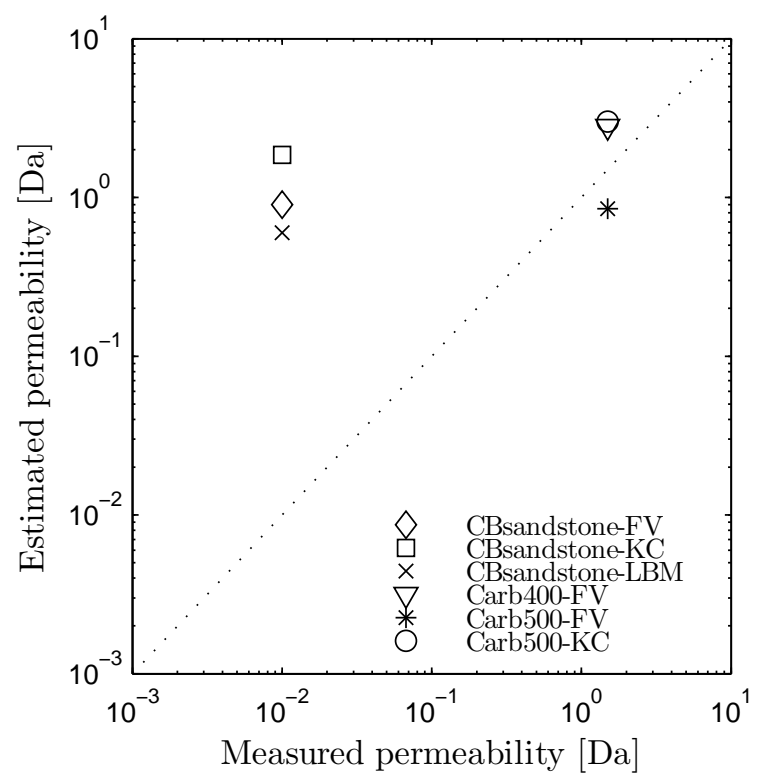

Figure 14. Comparison of different permeability estimates

rays $\mu-C T$ images is another source of uncertainty and might influence the permeability. However, despite ongoing developments and recent introduction of new algorithms, the segmentation of X-rays $\mu-C T$ images of porous materials is still a controversial research field, and there is no evidence that more advanced methods are better than global thresholding for fluid flow modelling (see Iassonov et al. (2009); Wildenschild and Sheppard (2013) for more details on this subject).

Finally, the $\mathrm{KC}$ equation fitted with the specific surface area value estimated using statistical correlation gives results close to the numerical ones for both rock formations. However, it must be noted that eq. (10) relies on the constants $m_{1}$ and $m_{2}$, whose calibration depends upon the morphological features of the rock. For example, for the sample CBsandstone3, using the range of reference values for $m_{1}$ and $m_{2}$ given in literature (Archie et al., 1942; Walsh and Brace, 1984) produced permeability values ranging from 1230 to $2360 \mathrm{mDa}$. The lower limit is very close to the average numerical permeability of $940 \mathrm{mDa}$. A perfect fit could be obtained for values of $m_{1}$ and $m_{2}$ equal to 3.1 and 2.2 respectively, or, working on $m_{2}$ only, to 3 and 2.22. This latter result also shows how eq. (10) is more sensitive to $m_{2}$ than $m_{1}$. This is because $m_{2}$ is related to the formation factor, which is a key quantity describing the pore-structure of a rock, while $m_{1}$ depends on the shape and roughness of the pores, and can always be reasonably taken as equal to its upper limit. For the carbonate, the analysis is similar. For the sample Carb400, using the reference values of $m_{1}$ and $m_{2}$ yielded a range of permeability values from 3500 to $7590 \mathrm{mDa}$. Again the lower limit is closest to the average numerical permeability of $2800 \mathrm{mDa}$, and the fit is obtained for values of $m_{1}$ and $m_{2}$ equal to 3 and 2.12 respectively.

\section{Bulk momentum balance and a new covergence criterion for the SIMPLE algorithm}

The traditional convergence criterion for the SIMPLE algorithm utilizes the continuity imbalance, i.e. the r.h.s. of the artificially built pressure-correction equation, as a residual. It is then demanded that its normalization to an appropriate quantity be less than a given tolerance (Patankar, 1980; Perić et al., 1988). For example, Mostaghimi et al. (2013) proposed as a convergence criterion to check the 
normalized continuity imbalance (NCI), defined as the ratio of the average continuity imbalance $\bar{b}_{c}$ over the average flow rate $\bar{Q}$ as follows:

$$
N C I=\frac{\bar{b}_{c}}{\bar{Q}},
$$

and to terminate the computation when NCI is less than a given tolerance $\lambda$. Alternatively, a general stopping criterion based on the reduction of the residual can be used (Ferziger and Peric, 2012). In this case, iterations are stopped when the average residuals of all equations, namely pressure-correction and $\mathrm{x}, \mathrm{y}, \mathrm{z}$ momentum, are reduced to a given fraction of its original size. However, both these criteria are based on local satisfaction of the governing equations, and no information is provided at the macroscopic level. The latter aspect is of particular interest for pore-scale modelling of reservoir rocks, especially when one wants to use the pore-scale results for upscaling over REVs, as in case of permeability estimation. Darcy's law, which is used here, is indeed none other than expression of the macroscopic momentum balance (Whitaker, 1986).

Let us write the momentum equation (13) integrated over a control volume $\mathrm{V}$ having boundary area $A$ as follows:

$$
\mu \int_{A} \frac{\partial u_{j}}{\partial x_{i}} n_{i} d A-\int_{A} p n_{j} d A+B=0,
$$

where $\mathrm{n}$ is the unit normal pointing outwards the surface and $\mathrm{B}$ accounts for body forces. Considering the whole bulk medium as control volume, each of the two areal integrals in eq. (18) can be decomposed into two sub-integrals: one over the external boundary pore area $A_{p}$ and one over the internal pore-grain interfacial area $A_{s}$. The momentum equations for the whole medium can therefore be written as

$$
\mu \int_{A_{p}} \frac{\partial u_{j}}{\partial x_{i}} n_{i} d A-\int_{A_{p}} p n_{j} d A+\mu \int_{A_{s}} \frac{\partial u_{j}}{\partial x_{i}} n_{i} d A-\int_{A_{s}} p n_{j} d A+B=0 .
$$

The first term in eq. (19) is zero because of the applied boundary conditions (zero velocity-gradient at boundaries where pressure is prescribed and symmetry at the other four faces). The second term is the macroscopic force due to the applied pressure difference $\Delta P$. The third and fourth terms are usually referred to as the viscous (or friction) drag $D_{v}$ and the pressure (or form) drag $D_{p}$ respectively. Eq. (19) in its compact form $\Delta P+B=D_{p}+D_{v}$ represents a balance of forces at the macroscopic level and must be satisfied by the solution of the Stokes equations (12)-(13).

Hence, the proposed convergence criterion consists on monitoring the Relative Error in the Bulk Momentum Balance (REBMB) defined as

$$
R E B M B=\left|\frac{\Delta P+B-\left(D_{p}+D_{v}\right)}{\Delta P+B}\right| .
$$

This new criterion has the major merit of giving a clear physical interpretation of the approximate solution, i.e. how close one is to satisfy the bulk momentum balance and therefore how confident one can be in using Darcy's law for predicting the absolute permeability. This makes the criterion particularly useful for pore-scale modelling of reservoir rocks.

To test the new convergence criterion, we performed a test on the sample CBsandstone1b. Results are shown in Fig. 15. We monitored four quantities: the normalized average residuals of the momentum 


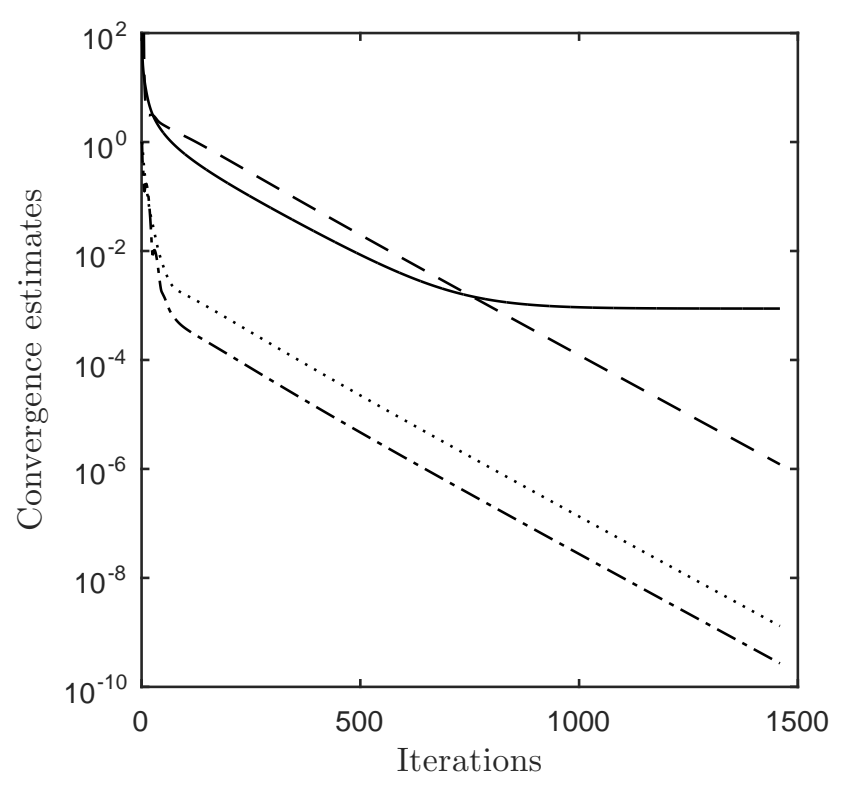

Figure 15. Comparison of different convergence indicators as a function of the outer iterations in the SIMPLE algorithm. Solid line: REBMB computed using eq. (20). Dashed line: NCI computed using eq. (17). Dotted line: normalized average residual of the momentum component in the direction of the flow. Dash-dot line: normalized average residual of the pressure-correction equation

component in the flow direction and pressure-correction equations, the normalized continuity imbalance, eq. (17), and the proposed REBMB, eq. (20). As the graph clearly shows, the normalized average residuals of both equations keep decreasing linearly as the solution of the respective PDE improves. NCI displays a similar behaviour. The REBMB instead converges to an asymptotic value as the macroscopic balance of forces in the flow direction can no longer be further refined with additional iterations. The achieved REBMB is less than $0.07 \%$ which can be considered an accurate measure. The fact that an asymptotic value is reached offers a valuable stopping criterion, provided that continuity must be satisfied as well. In light of this, the best convergence criterion appears to be a combination of the new one based on bulk momentum balance and the classical ones based on residual reduction. A suggested stopping criterion would be the scenario where the asymptotic value of REBMB is reached and the residuals have been reduced by 4-5 order of magnitude. Finally, it must be noted that this overall convergence criterion is only related to the quality of the final approximate solution and does not impact the optimum computing time, the latter being determined primarily by the method for the solution of the linear systems.

\section{Conclusions}

We presented here an integrated approach comprising computer tomography, statistical correlation and numerical simulations, for predicting the petrophysical properties, namely REV, permeability and mean pore size, of different reservoir rocks. We used the two-point spatial correlation function to analyze the pore-system and the FV-SIMPLE algorithm to solve the Stokes equations of flow. By analyzing several binarized small samples (order of $\mathrm{mm}$ ) scanned at a resolution down to $5 \mu \mathrm{m}$, we showed the potential of this approach to provide thorough characterization of reservoir rocks. The two-point spatial correlation function was shown to be an effective way for estimating REV and REA, as well as a powerful method for evaluating the mean pore and grain sizes. Using these tools, pore sizes of $70-90 \mu \mathrm{m}$ for the 
sandstone and 120-260 $\mu \mathrm{m}$ for the carbonate were estimated, in good agreement with the respective values of $100 \mu \mathrm{m}$ and from $90 \mu \mathrm{m}$ to $700 \mu \mathrm{m}$ measured from high resolution SEM images. For the carbonate, comparison of grain size was also made, showing good agreement between the statistically estimated values of 240-380 $\mu \mathrm{m}$ and the morphological data of 200-480 $\mu \mathrm{m}$ obtained using a standard petrogtaphic microscope. Rock homogeneity and anisotropy were also effectively evaluated using spatial correlation tools. This can be very helpful when selecting the most representative sample for use in more sophisticated numerical analyses. Moreover, this approach could be used to link the configuration of the pore system to the petrophysical properties of rocks, in a similar way to what Kozeny-Carman relationships do, e.g. the heterogeneity of the carbonate pore-system reflects on the variability in the predicted permeability for different sizes of the sub-samples. Comparison of all the available permeability estimates revealed good agreement between different numerical estimates, i.e. FV and LBM, but a discrepancy with the experimentally measured value. The latter is probably due to the difference in scale of the samples, resulting in significant changes in connectivity and tortuosity. For the sandstone, this discrepancy is larger due to the complexity of the pore system, i.e. the presence of sub-micro imperfections that cannot be detected by the resolution used to resolve the pore-space. More samples from different rock formations therefore need to be studied in order to establish a relationship between permeability estimates at different scales. This can be subject of a future more comprehensive study.

Finally, we presented a new convergence criterion for the SIMPLE algorithm, and more generally for the family of pressure-correction methods, based on the bulk momentum balance. This criterion provides information into significant fluid mechanics quantities such as form and friction drags, and is particularly useful for pore-scale modelling of reservoir rocks, especially when the predicted flow quantities are used for upscaling, e.g. Darcy's law. Moreover, the distribution of the error as a function of the iterations showed an asymptotic behaviour. This provides a valuable stopping criterion when the new convergence indicator is effectively used in combination with classical normalized residuals.

\section{Acknowledgements}

This work was jointly sponsored by EPSRC (EP/I010971/1) and NSFC China.

Adler, P., Jacquin, C., and Quiblier, J. (1990). Flow in simulated porous media. International Journal of Multiphase Flow, 16(4):691-712.

Al-Omari, A. and Masad, E. (2004). Three dimensional simulation of fluid flow in x-ray ct images of porous media. International Journal for Numerical and Analytical Methods in Geomechanics, 28(13):13271360.

Archie, G. E. et al. (1942). The electrical resistivity $\log$ as an aid in determining some reservoir characteristics. Transactions of the AIME, 146(01):54-62.

Bakke, S., Øren, P.-E., et al. (1997). 3-d pore-scale modelling of sandstones and flow simulations in the pore networks. Spe Journal, 2(02):136-149. 
Bear, J. (1972). Dynamics of fluids in porous media.

Berryman, J. G. (1985). Measurement of spatial correlation functions using image processing techniques. Journal of Applied Physics, 57(7):2374-2384.

Berryman, J. G. and Blair, S. C. (1986). Use of digital image analysis to estimate fluid permeability of porous materials: Application of two-point correlation functions. Journal of applied Physics, 60(6):1930-1938

Blunt, M. J., Bijeljic, B., Dong, H., Gharbi, O., Iglauer, S., Mostaghimi, P., Paluszny, A., and Pentland, C. (2013). Pore-scale imaging and modelling. Advances in Water Resources, 51:197-216.

Blunt, M. J., Jackson, M. D., Piri, M., and Valvatne, P. H. (2002). Detailed physics, predictive capabilities and macroscopic consequences for pore-network models of multiphase flow. Advances in Water Resources, 25(8):1069-1089.

Chen, S. and Doolen, G. D. (1998). Lattice boltzmann method for fluid flows. Annual review of fluid mechanics, 30(1):329-364.

Clavaud, J.-B., Maineult, A., Zamora, M., Rasolofosaon, P., and Schlitter, C. (2008). Permeability anisotropy and its relations with porous medium structure. Journal of Geophysical Research: Solid Earth, 113(B1).

Coelho, D., Thovert, J.-F., and Adler, P. (1997). Geometrical and transport properties of random packings of spheres and aspherical particles. Physical Review E, 55(2):1959.

Dong, H. (2008). Micro-CT imaging and pore network extraction. PhD thesis, Department of Earth Science and Engineering, Imperial College London.

Ferziger, J. H. and Peric, M. (2012). Computational methods for fluid dynamics. Springer Science \& Business Media.

Frisch, U., Hasslacher, B., and Pomeau, Y. (1986). Lattice-gas automata for the navier-stokes equation. Physical review letters, 56(14):1505.

Guibert, R., Nazarova, M., Horgue, P., Hamon, G., Creux, P., and Debenest, G. (2015). Computational permeability determination from pore-scale imaging: Sample size, mesh and method sensitivities. Transport in Porous Media, 107(3):641-656.

Haines, T. J., Michie, E. A., Neilson, J. E., and Healy, D. (2016). Permeability evolution across carbonate hosted normal fault zones. Marine and Petroleum Geology, 72:62-82.

Haines, T. J., Neilson, J. E., Healy, D., Michie, E. A., and Aplin, A. C. (2015). The impact of carbonate texture on the quantification of total porosity by image analysis. Computers \& Geosciences, 85:112-125.

Iassonov, P., Gebrenegus, T., and Tuller, M. (2009). Segmentation of x-ray computed tomography images of porous materials: A crucial step for characterization and quantitative analysis of pore structures. Water Resources Research, 45(9). 
Lamy-Chappuis, B. (2015). Mineral-Fluid interactions and their implications for the sequestration of CO2 in saline aquifers. PhD thesis, University of Leeds.

Lamy-Chappuis, B., Angus, D., Fisher, Q., Grattoni, C., and Yardley, B. W. (2014). Rapid porosity and permeability changes of calcareous sandstone due to co2-enriched brine injection. Geophysical Research Letters, 41(2):399-406.

Litster, S., Epting, W., Wargo, E., Kalidindi, S., and Kumbur, E. (2013). Morphological analyses of polymer electrolyte fuel cell electrodes with nano-scale computed tomography imaging. Fuel Cells, 13(5):935-945.

Martys, N. S., Torquato, S., and Bentz, D. (1994). Universal scaling of fluid permeability for sphere packings. Physical Review E, 50(1):403.

Mostaghimi, P., Blunt, M. J., and Bijeljic, B. (2013). Computations of absolute permeability on micro-ct images. Mathematical Geosciences, 45(1):103-125.

Øren, P.-E. and Bakke, S. (2002). Process based reconstruction of sandstones and prediction of transport properties. Transport in Porous Media, 46(2):311-343.

Oren, P.-E., Bakke, S., Arntzen, O. J., et al. (1998). Extending predictive capabilities to network models. SPE journal, 3(04):324-336.

Otsu, N. (1975). A threshold selection method from gray-level histograms. Automatica, 11(285-296):2327.

Patankar, S. (1980). Numerical heat transfer and fluid flow. CRC Press.

Perić, M., Kessler, R., and Scheuerer, G. (1988). Comparison of finite-volume numerical methods with staggered and colocated grids. Computers $\& 3$ Fluids, 16(4):389-403.

Politis, M., Kikkinides, E., Kainourgiakis, M., and Stubos, A. (2008). A hybrid process-based and stochastic reconstruction method of porous media. Microporous and Mesoporous Materials, 110(1):9299.

Sabharwal, M., Pant, L., Putz, A., Susac, D., Jankovic, J., and Secanell, M. (2016). Analysis of catalyst layer microstructures: From imaging to performance. Fuel Cells, 16(6):734-753.

Tashman, L., Masad, E., Crowe, C., and Muhunthan, B. (2003). Simulation of fluid flow in granular microstructure using a non-staggered grid scheme. Computers \& fluids, 32(9):1299-1323.

Valvatne, P. H., Piri, M., Lopez, X., and Blunt, M. J. (2005). Predictive pore-scale modeling of single and multiphase flow. In Upscaling Multiphase Flow in Porous Media, pages 23-41. Springer.

Walsh, J. and Brace, W. (1984). The effect of pressure on porosity and the transport properties of rock. Journal of Geophysical Research: Solid Earth (1978-2012), 89(B11):9425-9431. 
Whitaker, S. (1986). Flow in porous media i: A theoretical derivation of darcy's law. Transport in porous media, 1(1):3-25.

Wildenschild, D. and Sheppard, A. P. (2013). X-ray imaging and analysis techniques for quantifying porescale structure and processes in subsurface porous medium systems. Advances in Water Resources, $51: 217-246$.

Yeong, C. and Torquato, S. (1998). Reconstructing random media. Physical Review E, 57(1):495.

Zaretskiy, Y., Geiger, S., Sorbie, K., and Förster, M. (2010). Efficient flow and transport simulations in reconstructed 3d pore geometries. Advances in Water Resources, 33(12):1508-1516.

Zu, Y. and He, S. (2013). Phase-field-based lattice boltzmann model for incompressible binary fluid systems with density and viscosity contrasts. Physical Review E, 87(4):043301. 